\title{
Strong Enhancement of Light Extraction Efficiency in Sub-wavelength AIGaAs/GaAs Vertical-emitting Nanopillars
}

\author{
Bruno Romeira ${ }^{1 *}$, Jérôme Borme ${ }^{2}$, Hélder Fonseca ${ }^{3}$,João Gaspar ${ }^{3}$, and Jana B. Nieder ${ }^{1}$ \\ ${ }^{1}$ Dept. of Nanophotonics ; 2 Dept. of Quantum and Energy Materials ; ${ }^{3}$ Dept. of Micro and Nanofabrication, \\ INL - International Iberian Nanotechnology Laboratory, Av. Mestre José Veiga s/n, 4715-330 Braga, Portugal \\ *bruno.romeira@inl.int
}

Scaling down active nanophotonic devices, namely nano-lasers and nano-light-emitting diodes (nanoLEDs), to deep sub-micrometer sizes, is crucial to achieve small footprint $\left(<1 \mu \mathrm{m}^{2}\right)$, low energy consumption $(<10 \mathrm{fJ} / \mathrm{bit})$, and efficient $(>10 \%)$ light sources, as needed for future compact photonic integrated circuits for optical communications [1], and biosensing and bioimaging applications [2]. As the surface-to-volume ratio of these nanoscale sources increases substantially, among the numerous challenges, strong non-radiative processes and difficulties in extracting the light have been shown to have a detrimental effect on the external quantum efficiencies of nanoLEDs and nanolasers [3]. Although there has been intense research, particularly in light-enhancement and out-coupling methods, using for example 2D photonic crystals [4], optical nanoantennas [5], or nanowaveguides integrated with grating couplers [3], these approaches are extremely challenging to implement when the size of the light-emitting structures is drastically reduced to the deep-subwavelength $\left(<<\lambda^{3}\right)$ scale.

In this work, we report a strong enhanced signal at $\lambda \sim 670 \mathrm{~nm}$ in vertical-emitting undoped $\mathrm{AlGaAs} / \mathrm{GaAs} / \mathrm{AlGaAs}$ tapered pillars in a GaAs substrate, Fig. 1(a), when the emitting nominal area is decreased to the sub- $\mu \mathrm{m}$ scale. Vertical-emitting pillars ranging from $200 \mathrm{~nm}$ to $8 \mu \mathrm{m}$ lateral width were fabricated using ebeam lithography and dry etching techniques and characterized using a micro-photoluminescence (PL) microscope with $\lambda=561 \mathrm{~nm}$ laser excitation. Figure 1(b) shows examples of emission images for both optically pumped micropillars (top) and nanopillars (bottom) (the respective intensity profiles are shown inset). For the case of micropillars, clearly the light emission is reduced as the diameter decreases following a typical scaling law, $d^{2}$, of planar LEDs. However, as $d$ is reduced from $4 \mu \mathrm{m}$ to $0.2 \mu \mathrm{m}$ sizes, particularly in the range of $300 \mathrm{~nm}<d<400$ $\mathrm{nm}$, although the nominal emission area is reduced by a factor of more than 100 , the intensity is reduced only by $\sim 10$ times. For example, the emitting intensity peaks for pillars with $d=360 \mathrm{~nm}$, and the integrated intensity is comparable to pillars with $d \sim 1 \mu \mathrm{m}$ sizes. This strongly deviates from the $d^{2}$ dependence observed for micropillars, resulting in a 27-fold enhancement of emission. This striking effect is summarized in Fig. 1(c). Our FDTD simulations for a tapered $d=360 \mathrm{~nm}$ nanopillar, Fig. 1(a)(bottom), indicate this enhancement is a result of a 3-fold effect: i) suppression of optical modes due to lateral size reduction, ii) efficient out-coupling to air, and iii) more directed emission of tapered pillars. Notably, as shown in the blue circles of Fig. 1(c), the emission can be further improved after surface passivation with $\left(\mathrm{NH}_{4}\right)_{2} \mathrm{~S}$ and dielectric capping with a $\sim 50 \mathrm{~nm} \mathrm{SiO} \mathrm{S}_{2}$ layer. For the case of sub- $\mu \mathrm{m}$ pillars, a 3-fold improvement of light emission is achieved as compared with unpassivated samples.

In summary, a large improvement of light-extraction in sub- $\lambda$ vertical-emitting nanopillars is achieved. This pronounced effect enables bright emission in nanoscale devices comparable to the performance of $\mu \mathrm{m}$-sized devices. This result, combined with the suppression of surface recombination, is crucial for the future development of high-performance nanoscale optoelectronic devices for low-power optical interconnects, supporting the realization of room-temperature highly efficient light sources in photonic integrated circuits.
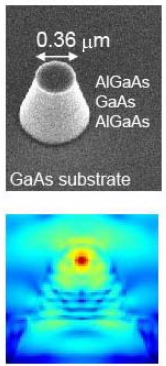

(a)

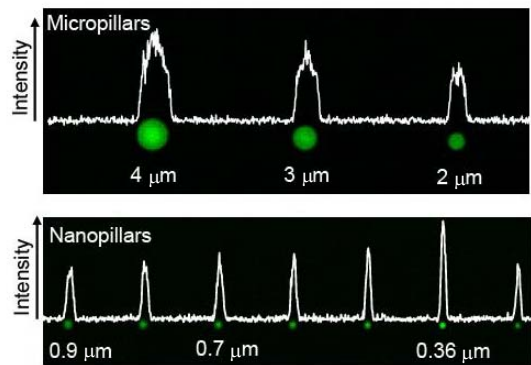

(b)

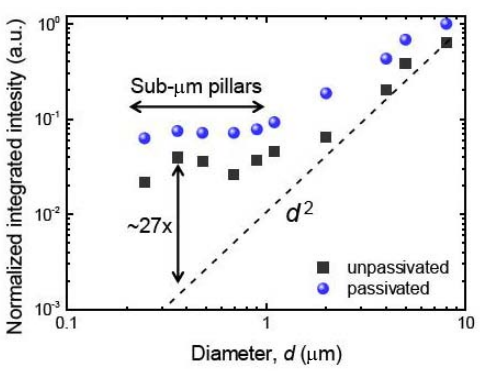

(c)

Fig. 1 (a) Scanning electron micrograph (SEM) image of a $\sim 0.36 \mu \mathrm{m}$ nanopillar (top), and finite-difference timedomain (FDTD) 3D simulations showing the field of a $\sim 0.36 \mu \mathrm{m}$ nanopillar with a $20^{\circ}$ tilted angle (bottom). (b) MicroPL images from vertical-emitting micro- and nano-pillars. Inset is shown the intensity profile. (c) Integrated intensity plot as a function of diameter for unpassivated and passivated samples (the dash line shows a $d^{2}$ dependence).

\section{References}

[1] D.A.B. Miller,"Attojoule Optoelectronics for Low-Energy Information Processing and Communications," J. Light. Technol. 35, 3 (2017).

[2] M. T. Hill and M. C. Gather, "Advances in small lasers," Nat. Phot. 8, 908 (2014).

[3] V. Dolores-Calzadilla, et al., "Waveguide-coupled nanopillar metal-cavity light-emitting diodes on silicon," Nat. Commun. 8, (2017).

[4] J. J. Wierer, et al., "III-nitride photonic crystal light-emitting diodes with high extraction efficiency," Nat. Phot. 3, 163 (2009).

[5] M. S. Eggleston, et al.” Optical antenna enhanced spontaneous emission,” Proc. Natl. Acad. Sci. 112, 6 (2015). 